\title{
Simple Approximation Algorithms and PTASs for Various Problems in Wireless Ad Hoc Networks
}

\author{
Xiang-Yang $\mathrm{Li}^{\mathrm{a}, 1}$, Yu Wang ${ }^{\mathrm{b}, *}$ \\ a Department of Computer Science, Illinois Institute of Technology, \\ 10 West 31st Street, Chicago, IL 60616, USA \\ ${ }^{\mathrm{b}}$ Department of Computer Science, University of North Carolina at Charlotte, \\ 9201 University City Blvd., Charlotte, NC 28223, USA
}

\begin{abstract}
A wireless ad hoc network is often composed of a set $V$ of $n$ wireless devices distributed in a two-dimensional domain. For each wireless device (also called node) $u \in V$, there is a transmission region within which signal-to-noise-ratio (SNR) is at least a threshold $\gamma$ so that the signal transmitted by $u$ can be correctly received by other nodes with high probability. The transmission region is often modeled as a disk centered at the node $u$. In addition, for each node $u$, there is an interference region within which the transmission from $u$ makes the signal-to-interference-andnoise-ratio (SINR) of the legitimate receiver smaller than the threshold $\gamma$ so that the legitimate receiver cannot correctly receive the message from the legitimate transmitter.

In this paper, we first present new graph models to model the communication graphs and the interference graphs defined by wireless ad hoc networks with attention to interference-free channel assignment or scheduling. Then we propose some simple approximation algorithms and/or PTASs (polynomial time approximation scheme) to approximate several classical graph problems such as maximum independent set, minimum vertex cover and minimum vertex coloring in these graph models. In addition, we also discuss various possible applications for these simple approximation algorithms and/or PTASs in wireless ad hoc networks.
\end{abstract}

Key words: Independent set, vertex cover, vertex coloring, disk graphs, PTAS, mobile ad hoc networks

* Corresponding author.

Email addresses: xli@cs.iit.edu (Xiang-Yang Li), ywang32@uncc.edu (Yu

Wang).

1 The author is partially supported by NSF CCR-0311174. 


\section{Introduction}

Wireless networking has received significant attention over the last few years due to its potential applications in various situations such as battlefield, emergency relief and so on. There are no wired infrastructures or cellular structures in wireless ad hoc networks. Multi-hop communication (carried out by relaying on intermediate nodes) is required when the receiver node is not within the sender's transmission range. Thus, each wireless node also acts as a router, forwarding data packets for other nodes.

A wireless ad hoc network is often composed of a set $u$ of $n$ wireless devices distributed in a two-dimensional domain. For each wireless device (also called node) $u \in V$, there is a transmission region within which signal-to-noise-ratio (SNR) is at least a threshold $\gamma$ so that the signal transmitted by node $u$ can be correctly received by other nodes with high probability. We assume that each wireless node has an omni-directional antenna, i.e., a single transmission of a node can be received by any node within a certain distance. Thus, the transmission region of $u$ is often modeled as a disk centered at the node $u$ with radius $t_{u}$. Here $t_{u}$ is called the transmission range of node $u$. When all wireless nodes have the same transmission radius, the communication network of a wireless ad hoc network is often modeled by unit disk graphs (UDG), in which two nodes are connected iff their Euclidean distance is no more than the transmission range. However, generally and practically, different wireless nodes will have different transmission range due to various tasks performed or different devices or different power capacities.

To increase the capacity of the network, frequency spectrum has to be reused as radio spectrum is one of the scarcest resources available. Interference is one of the major concerns in designing wireless networks. Signal-to-interference ratio is used to measure the quality of the received signal. It is defined as the ratio of the received signal strength from the intended source node to the received signal strengths from all other wireless nodes using the same frequency spectrum. The larger the signal-to-interference ratio, the better the quality of the wireless networks. Thus, it is often assumed in the literature that, for each node $u$, there is an interference region within which the transmission from $u$ makes the signal-to-interference-and-noise-ratio (SINR) of the legitimate receiver smaller than the threshold $\gamma$ so that the legitimate receiver cannot correctly receive the message from the legitimate transmitter. In other words, when a node $u$ transmits a signal, we assume that the signal in its interference region is strong enough to cause interference to every node $v$ inside the interference region. And it does not cause interference to nodes outside of the interference region. Obviously, it is assumed that the node $v$ intends to receive signal from some node $w$ other than $u$. Here we assume that no node which transmits on a certain frequency can, at the same time, receive on the 
same frequency. Thus, we assume an interference occurs if the transmission region of one node (node $w$ here) intersects with the interference region of another node (node $u$ here). See Figure 1 for illustrations. Most researchers, for

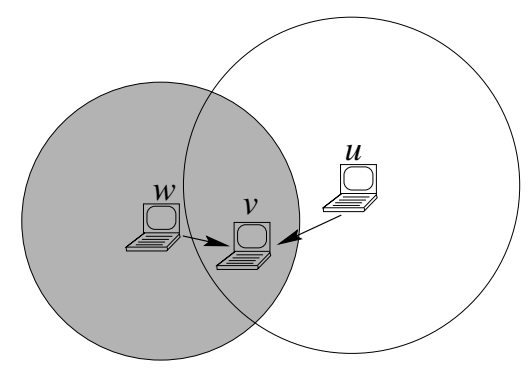

Fig. 1. Interference happens at node $v$ when the transmission region (denoted by the shaded disk) of node $w$ intersects with the interference region of node $u$.

simplicity, treat the transmission region of a node as its interference region. However, this simplification is not accurate in practice. Recently, more and more researchers have realized that the range of interference is larger than the transmission range [1-4]. For example, [1] uses a simple analytic model to show that in the open space environment, the interference range of a receiver is 1.78 times the transmitter-receiver distance. According to the IEEE 802.11 protocol implementation in the NS-2 network simulator, the interference range is more than two times the size of the communication range.

In this paper, we will propose a number of general graph models (namely, the general disk graph model, the interference graph model, the mutual communication graph model, the mutual-inclusion graph model and the conflict graph model) to model the communication networks or the interference graphs induced by a wireless ad hoc network by assuming that a node has an interference region larger than its transmission region. Notice that though many researchers have realized the large interference range, we are the first to model the underlying communication graph of ad hoc networks by considering the interference ranges. Some of our new graph models are similar to widely studied intersection graphs [5-7] in graph theory. Intersection graphs are graphs each of whose vertices is represented by a set, and two vertices are connected if their corresponding sets have a non-empty intersection. The example of intersection graphs includes interval graph [8], unit disk graph [9-11], and coin graph [12]. It is well-known that many classical NP-hard graph problems remain NP-hard even when they are restricted to intersection graph models. For example, the maximum independent set (MIS) problem, the minimum vertex cover (MVC) problem, and the minimum graph coloring (MGC) problem (also called minimum vertex coloring problem) remain NP-hard even when we restrict them to unit disk graphs [10]. Since UDG is a special case of graph models introduced here, constructing MIS remains NP-hard when restricted to these new graph models. Recently, Erlebach et al. [13] proposed a PTAS for MIS on traditional disk graphs based on the shifting strategy [14]. These classical graph problems 
have many potential applications in wireless ad hoc networks. For example, the solutions for MIS problem have been widely used for constructing virtual backbone for wireless ad hoc networks [15-21].

The main contributions of this paper are as follows: (1) We propose a number of new graph models to capture the communication characteristics or the interference characteristics of wireless ad hoc networks; (2) We present a simple approximation algorithm to compute a maximal independent set whose size is no more than a small constant times of the optimum in the new graph models; (3) We propose two PTASs to approximately solve the maximum weighted independent set problem and the minimum weighted vertex cover problem in the new graph models; (4) We show that the traditional simple greedy coloring method still achieves a constant approximation for minimum graph coloring problem in the new graph models. Notice that the methodologies of these simple algorithms and PTASs are not new but the analysis of their performance guarantees under the new graph models are not trivial extensions, which need to be carefully designed and proved.

The rest of the paper is organized as follows. In Section 2, we introduce our network models (intersection graphs) and three classical graph problems, then review previous results on these problems. We also discuss several possible applications of these problems and our proposed algorithms in mobile ad hoc networks. In Section 3, we show that the previous centralized method for computing MIS in disk graph model, when the transmission region is same with the interference region for every node $v$, still works in other models introduced in this paper. We also give a PTAS for approximating the maximum weighted independent set. In Section 4, we present algorithms suitable for approximating the minimum weighted vertex cover. Section 5 is devoted to study the approximation of graph coloring. We conclude our paper in Section 6 by pointing out some possible future research directions.

\section{Preliminaries}

\subsection{Wireless Ad Hoc Network Models}

We consider a wireless ad hoc network consisting of a set $V$ of $n$ wireless nodes distributed in a two-dimensional plane. In addition, we assume that the nodes are static or can be viewed as static during a reasonable period of time. Define the transmission radius of a node as the radius of the disk representing its transmission region. Similarly, we define the interference radius as the radius of the disk representing the interference region of this node. Each node $v \in V$ has a transmission radius $t_{v}$ and an interference radius $r_{v}$, where $0<t_{v} \leq r_{v}$. 
Let $D(v, r)$ denote the disk centered at $v$ with radius $r$. Each wireless node then defines two disks: the transmission disk $D\left(v, t_{v}\right)$ and the interference disk $D\left(v, r_{v}\right)$. The set of wireless nodes $V$ defines two sets of disks $\mathcal{T}=\left\{D\left(v, t_{v}\right) \mid\right.$ $v \in V\}$ and $\mathcal{D}=\left\{D\left(v, r_{v}\right) \mid v \in V\right\}$ in the two dimensional plane. Given two wireless nodes $u$ and $v$, define their intersection region $I(u, v)$ as follows:

$$
I(u, v)=\left(D\left(u, t_{u}\right) \cap D\left(v, r_{v}\right)\right) \bigcup\left(D\left(u, r_{u}\right) \cap D\left(v, t_{v}\right)\right) .
$$

See Figure 2 for an illustration of intersection regions.

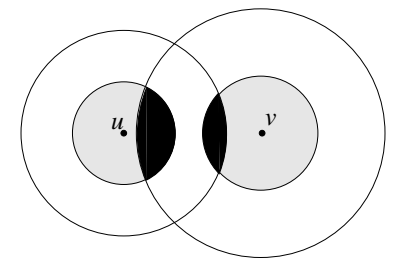

Fig. 2. The black region denotes the intersection region $I(u, v)$. The shaded disks are the transmission disks and the non-shaded disks are the interference disks.

We then introduce several intersection graph models to model wireless ad hoc networks.

Disk graphs has been widely studied. Traditionally, a disk graph is the intersection graph of the set of disks each of which is centered at a unique node from a node set $V$, i.e., two nodes $u, v \in V$ are connected in the traditional disk graph if the two disks centered at $u$ and $v$ have a non-empty intersection. We first extend this conventional definition to wireless ad hoc networks where each wireless node defines two disks, namely, the interference disk and the transmission disk. Here, the disk graph (DG) for wireless ad hoc networks has an edge $u v$ if and only if the intersection area $I(u, v)$ is not empty. In other words, there is an edge $u v$ if and only if $\|u v\| \leq \min \left(t_{u}+r_{v}, r_{u}+t_{v}\right)$. Here $\|u v\|$ is the Euclidean distance between two wireless nodes $u$ and $v$. Notice that, the special case when $t_{u}=r_{u}$ for all wireless nodes $u$ was studied in [13,29] recently. Li et al. [29] also studied some other geometry graphs derived from wireless networks when every node $u$ has $t_{u}=r_{u}$.

In wireless ad hoc networks, if two nodes $u$ and $v$ are not connected in the disk graph, then they can transmit messages simultaneously without causing interference to each other under our interference model. Therefore, the chromatic number of the disk graph defined above is an upper bound of the minimum number of frequencies (also called channels) needed when we must assign a channel to each node so that all nodes can communicate simultaneously without interferences. Here we assume that a wireless node can tune its receiving device to different channels other than its transmission channel.

Obviously, the disk graph model is an over-estimation for interferences for ad 
hoc wireless networks. For example, when the intersection region $I(u, v)$ of two corresponding wireless nodes $u$ and $v$ is not empty, these two nodes can still use the same channel if the intersection region $I(u, v)$ does not contain any wireless node inside. To capture this property, we define the interference graph (IG) as follows: two nodes $u$ and $v$ are connected if and only if there is a node from $V$ inside their intersection region $I(u, v)$. The chromatic number of the interference graph is exactly the minimum number of channels needed when we must assign a channel to each node so that all nodes can communicate simultaneously without causing interferences in our interference model.

The interference graph model captures all links $(u, v)$ where $u$ and $v$ cannot transmit simultaneously using the same channel. In the actual wireless communications, two nodes $u$ and $v$ can communicate with each other directly if they are within the transmission range of each other. We call the graph formed by all such links $(u, v)$ as mutual communication graph (MCG). In other words, a link $u v$ is kept in the mutual communication graph if and only if there is a physical symmetric link $u v$. In the remainder of the paper, we will not study the approximation algorithms for MCG since this has been studied in [29]. For completeness, we also introduce two interesting new graph models here, namely, mutual-inclusion graph (MG) and conflict graph (CG). We believe that these two new graph models may also find some applications later in wireless ad hoc networks. In the mutual-inclusion graph, two nodes $u$ and $v$ are connected if and only if the intersection region $I(u, v)$ contains both $u$ and $v$ inside. While in the conflict graph model, two nodes $u$ and $v$ are connected if and only if the intersection region $I(u, v)$ contains at least one of $u$ and $v$ inside. Obviously, the chromatic number of the conflict graph is a lower-bound of the minimum number of channels needed when we must assign a channel to each node so that all nodes can communicate without interferences.

By definition, $M C G \subseteq M G \subseteq C G \subseteq I G \subseteq D G$.

\subsection{Graphs Problems and Their Applications}

A subset of vertices in a graph $G$ is an independent set (IS) if for any pair of vertices, there is no edge between them. It is a maximal independent set if no more vertices can be added to it to form a larger independent set. It is a maximum independent set (MIS) if no other independent set has more vertices. The goal of the MIS problem is to compute, given a graph, a subset of pair-wisely unconnected vertices with the maximum cardinality.

Given a graph $G=(V, E)$, a subset $V^{\prime} \subseteq V$ of vertices is a vertex cover if for every edge in $E, V^{\prime}$ contains at least one of its end-vertices. In other words, every edge is dominated by some node from $V^{\prime}$. The goal of minimum vertex 
cover (MVC) problem is to find a vertex cover with the minimum cardinality.

The graph coloring (GC) problem (often called vertex coloring problem) is to assign each vertex a color. A vertex coloring is valid if two adjacent vertices are assigned different colors. The minimum graph coloring (MGC) problem is to use minimum number of colors so a valid vertex coloring can be obtained.

These three problems are classical graph optimization problems. It has been proved that they all are NP-hard even under the traditional intersection graph model. The algorithms to solve these classical graph problems can be widely used for different applications in wireless networks. For example, the algorithms for the maximum independent set have been used as clustering algorithms for wireless ad hoc networks [15-22]; the algorithms for the minimum vertex coloring have been applied to solve the channel assignment problems $[23,24]$ and packet scheduling [25-28] in wireless ad hoc networks. In Subsection 2.5, more possible applications of these problems for wireless ad hoc networks are discussed in detail. Therefore, in this paper, we are interested in designing efficient algorithms to approximate the maximum independent set, the minimum vertex cover, and the minimum graph coloring for the graph models defined above. These efficient algorithms will help us solving several hard (NP-complete) optimization problems pertaining to QoS- and energyaware dynamic wireless ad hoc network infrastructures, besides routing, and medium access mechanisms.

Given a graph $G$ of $n$ vertices and a problem $P$, let $O P T_{P}(G)$ denote an optimum solution of problem $P$ when the input graph is $G$. For a maximization problem $P$ (such as maximum independent set), an algorithm is a $\rho$-approximation algorithm for problem $P$ if, given any input graph $G$, it runs in polynomial time and always computes a solution that is at least $\frac{1}{\rho} O P T_{P}(G)$. For a minimization problem $P$ (such as minimum vertex cover), an algorithm is a $\rho$-approximation algorithm for problem $P$ if, given any input graph $G$, it runs in polynomial time and always computes a solution that is at most $\rho \cdot O P T_{P}(G)$. An algorithm is a polynomial-time-approximation-scheme (PTAS) if, for any additional parameter $\varepsilon>0$, it runs in time polynomial of $n$ and always computes a solution that is at least $\frac{1}{1+\varepsilon} O P T_{P}(G)$ for a maximization problem $P$ and at most $(1+\varepsilon) \cdot O P T_{P}(G)$ for a minimization problem $P$.

\subsection{Previous Results}

For unit disk graphs, Marathe et al. [11] gave simple centralized heuristics to approximate the maximum independent set, the minimum vertex cover, the minimum vertex coloring, the minimum dominating set, and the mini- 
mum connected dominating set within constant $3, \frac{3}{2}, 3,5$, and 10 respectively. Hunt et al. [30] then presented the first PTASs to approximate the maximum independent set, the minimum vertex cover, and the minimum dominating set in UDG. Recently, Wang et al. [22] proposed a distributed algorithm to approximate maximum weighted independent set for wireless networks.

For traditional disk graphs, it was claimed in [11] that the MIS problem can be approximated within 5. Then Erlebach et al. [13] proposed an elegant PTAS for maximum weighted independent set (MWIS) and the maximum weighted vertex cover (MWVC) based on the shifting strategy proposed by Hochbaum [14]. The algorithm runs in time $\frac{1}{\varepsilon^{2}} n^{O\left(\frac{1}{\varepsilon^{4}}\right)}$. Thus, when each node $u$ has the same transmission range $t_{u}$ and the interference range $r_{u}$, we already have PTASs for both MWIS and MWVC problems in the disk graph model.

Recently, Li et al. [29] also studied the MWIS and MWVC problems under the following graph models derived from wireless ad hoc networks: interference graph IG, mutual-inclusion graph MG, and the conflict graph CG when the interference region of every node is the same as its transmission region. Both simple heuristics with constant bounded approximation ratios and PTASs are given for the maximum weighted independent set and the minimum weighted vertex cover under the graph models DG, IG, MG, and CG. Simple heuristics with constant approximation ratios are presented for the minimum graph coloring problems over all graph models.

\subsection{Our Results}

In this paper, we present efficient simple approximation algorithms to solve MIS, and MGC under all graph models (disk graphs, interference graphs, mutual-inclusion graphs, mutual communication graphs, and conflict graphs) derived from wireless ad hoc networks. We then present PTASs for the MWIS and MWVC problems for all graph models introduced in this paper. Our simple constant approximation algorithms need only every node's interference radius and the underline graph structure, but no transmission radius and the geometry location of each node are needed. Our PTASs need the exact geometry location and the interference radius of each node instead. We present PTASs for MIS and MVC when the network is modeled by graph models DG, IG, MG, and CG. We also show that the graph coloring problem in these graph models can be approximated with 5 for mutual-inclusion graphs, mutual communication graphs, and conflict graphs and 40 for disk graphs and interference graphs. Notice that, when $r_{v}=t_{v}$ for every node $v$, the graph coloring problem in DG can be approximated within 5 using a simple heuristic [29]. However, when $r_{v} \neq t_{v}$, this simple heuristic cannot guarantee approximation ratio 5 anymore; instead, we show that it is at most 40 and at 
least 12 by giving an example.

\subsection{Applications in Wireless Ad Hoc Networks}

The problems (maximum independent set, minimum vertex cover and minimum graph coloring) we will study are three classical graph problems. They have been used to model several optimization problems in different applications in wireless networks.

Cluster technique has been widely used in wireless ad hoc network. It is one popular way to avoid flooding in the whole networks by restricting the communications to only be in a backbone formed by a subset of wireless nodes. Clustering method selects a subset of wireless nodes as clusterheads, and forms a hierarchical structure like Internet to perform the communications. Usually, the hierarchical structure (often called virtual backbone) is a connected dominating set. Many connected dominating set based (or cluster based) routing protocols have been proposed [17,18,34-36]. Efficient algorithms for constructing connected dominating sets in ad hoc wireless networks were well studied [15-22]. Several of them used the maximum independent set as the first step in their algorithms to select clusterheads. For example, in [20,21], they first built a maximal independent set by an MIS algorithm based on node IDs, then select several connectors to connect the nodes in the maximal independent set to a connected dominating set. Since they used the unit disk graph model, they proved that the maximal independent set constructed in the first step of their algorithm is at most 5 times the optimum. Therefore, when we use the intersection graphs to model the communications in the networks, we can apply our MIS heuristic or PTAS to build MIS and form the hierarchical cluster structure.

Notice that our PTAS actually approximates the maximum weighted independent set in which every node has a generic weight. Here the weight of each node could be defined by various practical applications. For example, the weight may represent the fitness or priority of each node to be a clusterhead. The lower weight means the higher priority. In practice, the weight could represent the power consumption rate of this node, the non-robustness of this node or a function of its security level. Recently, many proposed clustering algorithms [16,37-44] also considered different weights (such as node-degree, transmission power, stability or mobility and the battery power of the nodes) as a priority criterion to decide whether a node will be a clusterhead.

Beside forming the backbone for routing or broadcasting, the maximum independent set algorithms can also be used in other applications. For example, Zheng et al. [45] studied the time indexing problem in sensor networks. To 
enable time-indexed in-network storage of sensor data, they selected a subset of sensors, i.e., rendezvous points to collect, compress and store sensor data from its neighborhood for pre-defined periods of time. To consider the energy and storage balancing, we can apply our maximum independent set algorithms to select the rendezvous points. Another example, in [46], a simple cluster algorithm is used for selecting the wireless agents to perform intrusion detection in wireless ad hoc networks. We can also apply our method to their intrusion detection system to achieve more robust and power efficient agent selection.

Recently, Wan et al. [47] showed another application for maximum independent set in OVSF-CDMA (Orthogonal Variable Spreading Factor - Code Division Multiple Access) wireless ad hoc networks. In contrast to the conventional orthogonal fixed-spreading-factor CDMA code, OVSF-CDMA code consists of an infinite number of codewords with variable rates but not every pair of codewords are orthogonal to each other. In an OVSF-CDMA wireless ad hoc network, a code assignment has to be conflict-free, i.e., two nodes $u$ and $v$ can be assigned the same codeword or two non-orthogonal codewords if and only if neither of them is within the interference range of the other and no other node is located in the intersection region $I(u, v)$. In [47], the authors proposed several conflict-free CDMA/OVSF code assignment algorithms for wireless ad hoc networks when the interference region of every node is same as its transmission region. Their algorithms can achieve the total throughput or minimum rate within a constant factor of the optimum. All of their approximation algorithms applied some maximal independent set algorithms as part of their methods. If we would like to consider the non-identical transmission and interference ranges, we can apply our proposed MIS algorithm to do the code assignment for OVSF-CDMA wireless ad hoc network.

Vertex cover problem also has been studied and applied in research of wireless networks [48-50]. It was used for proving the NP-hardness of some optimization problems, such as minimum energy broadcast [48,49]. In [50], Zheng et al. studied wakeup mechanism for ad hoc networks to save energy in ad hoc networks. A wakeup mechanism associates each node with a slot schedule of length, termed as the wakeup schedule function (WSF). The authors showed the asymmetric WSF design problem is related to the vertex covering problem.

The last problem (minimum graph coloring or often called minimum vertex coloring) was also applied in wireless ad hoc networks to solve channel assignment or traffic scheduling problems [23-28,51,52]. In [27], Negi and Rajeswaran derived a bound on MAC (Medium Access Control) performance based on their physical layer model, by specifying a graph coloring heuristic. In [28], Tseng, Wu and Chen proposed a scheduling method called maximum traffic scheduling for IEEE 802.15.3. Their scheduling method applied our graph coloring method in [29] to finding the chromatic number. Both [25] and [26] also studied packet scheduling problem in wireless ad hoc networks by 
using coloring techniques. In addition, it is also well-known that coloring algorithms of graphs are applicable in channel assignment algorithms in wireless networks. For example, both [23] and [24] treated the channel assignment as a coloring problem and proposed different solutions. A distributed vertex coloring method was also used in the method proposed in [51] for constructing connected dominating sets for ad hoc networks. In [52], the authors proposed linear programs to output the bound of the power rate function of wireless ad hoc networks, where they also applied vertex coloring method on a usageconflict-graph.

\section{Maximum Independent Set}

In this section, we first present an algorithm that is easy to implement and approximates the MIS within 5 for mutual-inclusion graphs, mutual communication graphs and conflict graphs, and 40 for the disk graphs and the interference graphs introduced here. We next present a PTAS for the MIS problem in all graph models introduced in this paper by extending the methods presented in $[13,29]$.

\subsection{Simple Approximation Method}

Assume that we know the interference radius $r_{v}$ of each wireless node $v$. In addition, we also know the graph representation of the underlying graph, which could be DG, MG, IG, or CG. If the graph structure is unknown, then we need the transmission range $t_{v}$ and the exact geometry location of each node $v$ to construct the corresponding graph.

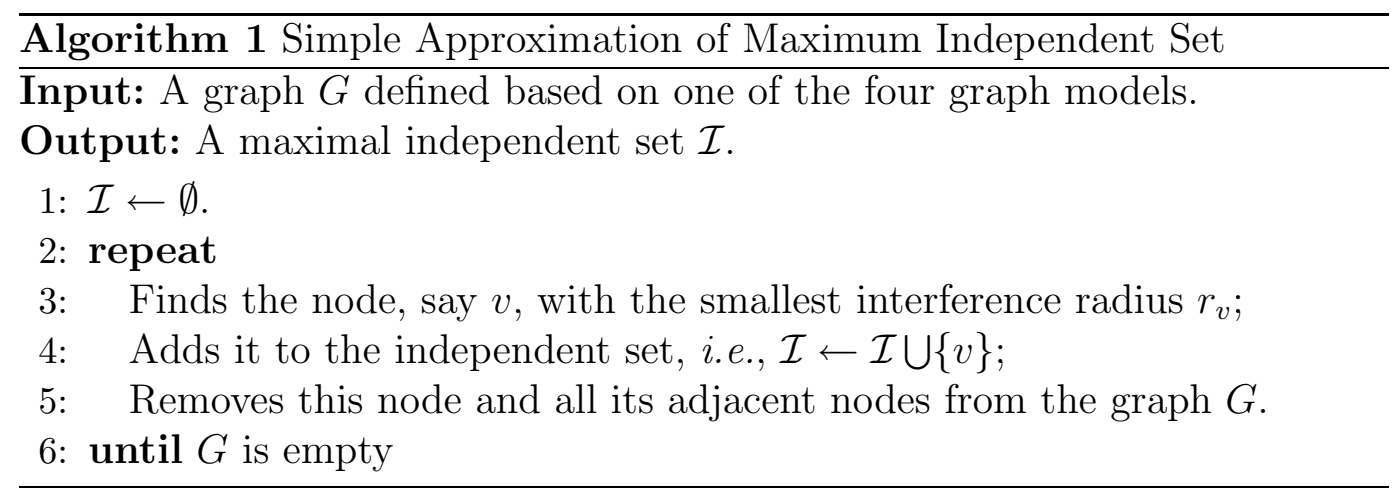

Obviously, this algorithm does compute a maximal independent set with $O(n \log n)$ running time. We then prove the following theorems that guarantee the quality of the computed independent set. Let $\mathcal{I}$ be the computed independent set of nodes. 
Theorem 1 The computed $\mathcal{I}$ has size at least $\frac{1}{5}$ of that of MIS if the input graph is a mutual-inclusion graph or a conflict graph.

Proof. Notice that the independent set $\mathcal{I}$ computed by our algorithm is maximal. Therefore, every node in an optimum MIS solution is either in $\mathcal{I}$ or is connected to some node from $\mathcal{I}$. We claim that, for any node $u$ in $\mathcal{I}$, there are at most 5 nodes from an optimum MIS solution such that they are connected to $u$ and are removed by the algorithm due to the removing of $u$. This immediately implies our theorem.

To prove our claim, we show that if $v_{1}$ and $v_{2}$ are both from an optimum solution, are connected to $u$ and removed by $u$, then $\angle v_{1} u v_{2}>\frac{\pi}{3}$. Remember that the selection of node $u$ always implies that $r_{u} \leq r_{v_{i}}$ for $i=1,2$. We prove $\angle v_{1} u v_{2}>\frac{\pi}{3}$ for different input graph models separately.

If the underlying graph is a mutual-inclusion graph, $u v_{i}$ is an edge implies that $I\left(u, v_{i}\right)$ contains both nodes $u$ and $v_{i}$. Then obviously node $v_{i}$ is inside $D\left(u, r_{u}\right)$. Thus, $\left\|u v_{i}\right\| \leq r_{u}$ for $i=1,2$. Moreover, because $v_{1}$ and $v_{2}$ are independent, we have either $v_{1}$ is not inside $D\left(v_{2}, r_{v_{2}}\right)$ or $v_{2}$ is not inside $D\left(v_{1}, r_{v_{1}}\right)$ or both. It implies that $\left\|v_{1} v_{2}\right\|>\min \left(r_{v_{1}}, r_{v_{2}}\right) \geq r_{u}$. Thus, $\left\|v_{1} v_{2}\right\|>\left\|u v_{i}\right\|$ for $i=1,2$. In other words, $\angle v_{1} u v_{2}>\frac{\pi}{3}$.

If the underlying graph is a conflict graph, $u v_{i}$ is an edge implies that $I\left(u, v_{i}\right)$ contains at least one of the nodes $u$ and $v_{i}$. It is easy to show that node $u$ is inside $D\left(v_{i}, r_{v_{i}}\right)$ from $r_{u} \leq r_{v_{i}}$. Thus, we have $\left\|u v_{i}\right\| \leq r_{v_{i}}$ for $i=1,2$. Since $v_{1}$ and $v_{2}$ are independent, node $v_{1}$ is not inside $D\left(v_{2}, r_{v_{2}}\right)$ and node $v_{2}$ is not inside $D\left(v_{1}, r_{v_{1}}\right)$. Thus, $\left\|v_{1} v_{2}\right\|>\max \left(r_{v_{1}}, r_{v_{2}}\right)$. Consequently, $\left\|v_{1} v_{2}\right\|>\left\|u v_{i}\right\|$ for $i=1,2$. In other words, $\angle v_{1} u v_{2}>\frac{\pi}{3}$.

This finishes the proof of the theorem. Notice that, in the above proof, surprisingly, the transmission radii of the nodes do not play any role.

In case of DG and IG graph models, the following theorem still guarantees a constant approximation ratio for MIS.

Theorem 2 The computed $\mathcal{I}$ has size at least $\frac{1}{40}$ of that of MIS if the input graph is a disk graph or an interference graph.

Proof. We prove this using an area argument. Consider any node $u$ from $\mathcal{I}$ selected by our algorithm. The nodes from an optimum solution, which are connected to $u$ and removed by $u$, can be partitioned into two cases: outside $D_{u}$ or inside $D_{u}$. Remember that here $D_{u}=D\left(u, r_{u}\right)$ in our notations.

First consider the nodes outside $D_{u}$. Let $v_{1}, v_{2}, \cdots, v_{k} \notin D_{u}$ be the $k$ nodes from the optimum solution that are connected to $u$ and are removed by the algorithm due to the removing of $u$. The selection of $u$ implies that $r_{v_{i}} \geq r_{u}$ 
for all $i=1,2, \cdots, k$.

Node $v_{i}, i=1,2, \cdots, k$ is connected to $u$ implies that $D_{i}=D\left(v_{i}, r_{v_{i}}\right)$ intersects with disk $D_{u}=D\left(u, r_{u}\right)$ because if they do not intersect then obviously $I\left(u, v_{i}\right)$ is empty, which further implies that there is no edge $u v_{i}$ in any graph models introduced here. In addition, the disk $D_{i}=D\left(v_{i}, r_{v_{i}}\right)$ centered at $v_{i}, i=$ $1,2, \cdots, k$ cannot contain any node $v_{j}, j \neq i$ inside because all disks centered at $v_{i}, i=1,2, \cdots, k$, are mutually independent in the corresponding graph model (the disk graph or the interference graph model). If $v_{j}$ is inside $D_{i}=$ $D\left(v_{i}, r_{v_{i}}\right)$, then $I\left(v_{i}, v_{j}\right)$ contains $v_{j}$ regardless of the transmission radii of $v_{i}$ and $v_{j}$, implying that $v_{i} v_{j}$ is an edge in both the disk graph model and the interference graph model.

Let $B_{u}$ be the disk centered at $u$ with radius $2 r_{u}$ and $T_{u}=B_{u}-D_{u}$. Then every disk $D_{i}, i=1,2, \cdots, k$, intersect $T_{u}$ since it intersects $D_{u}$. It is not difficult to show that $T_{u} \cap D_{i}$ achieves the smallest area when $v_{i}$ is on the boundary of $B_{u}$ and $r_{v_{i}}=r_{u}$. See the left figure of Figure 3 . We can show that $\angle w v_{2} u>\frac{5}{12} \pi$. Thus, the smallest area is at least $\frac{5}{12} \pi r_{u}^{2}$. Notice that the area of $T_{u}$ is $3 \pi r_{u}^{2}$.
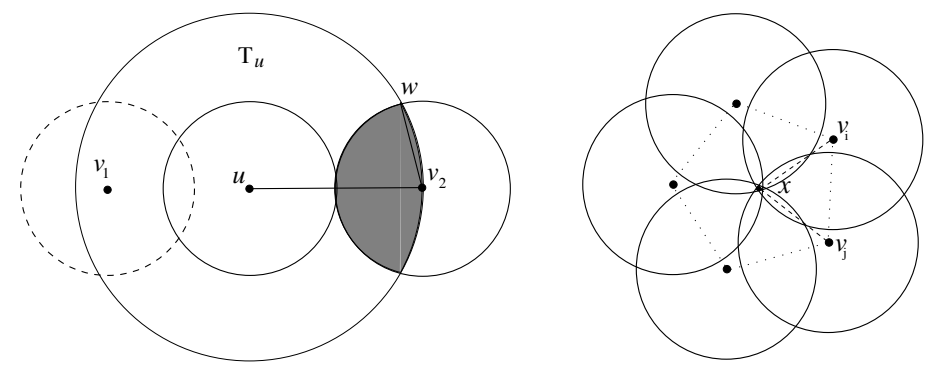

Fig. 3. Left: The intersection $T_{u} \cap D_{i}$ is bounded from below. Right: The number of independent disks which cover any point $x$ is bounded by 5 .

Notice that the region $T_{u} \cap D_{i}$ and $T_{u} \cap D_{j}$ for $1 \leq i, j \leq k$ may overlap. However, we will show that every point $x$ is covered by at most 5 disks from $D_{i}, i=1,2, \cdots, k$. See the right figure of Figure 3. Assume node $x$ is covered by two disks $D_{i}$ and $D_{j}$, i.e., $\left\|x v_{i}\right\| \leq r_{v_{i}}$ and $\left\|x v_{j}\right\| \leq r_{v_{j}}$. Then $\left\|v_{i} v_{j}\right\|>$ $\max \left(r_{v_{i}}, r_{v_{j}}\right)$ because $D_{i}$ and $D_{j}$ are independent in the corresponding graph model (DG or IG), which implies that $\angle v_{i} x v_{j}>\frac{\pi}{3}$. Thus, $x$ is covered by at most 5 independent disks. Therefore, by an area argument, we have $k \cdot \frac{5}{12} \pi r_{u}^{2}<$ $5 \cdot 3 \pi r_{u}^{2}$. Thus, $k \leq 35$.

Then consider the nodes inside $D_{u}$. Let $v_{1}, v_{2}, \cdots, v_{h} \in D_{u}$ be the $h$ nodes from the optimum solution that are connected to $u$ and are removed by the algorithm due to the removing of $u$. Then obviously, all disks centered at $v_{i}$, $i=1,2, \cdots, h$ contain node $u$. Since node $u$ is covered by at most 5 disks from previous analysis, we have $h \leq 5$.

Consequently, at most $35+5=40$ independent nodes are removed when we 
remove all nodes adjacent to a node $u$ from $\mathcal{I}$ selected by our algorithm. This finishes the proof.

The above theorems actually show that the graph models introduced in this paper have hereditary property: there is a node with a constant bounded number of independent neighbors, and the subgraph by removing this node and its neighbors also does.

Figure 4 gives a configuration example such that there are 12 independent nodes that are removed in DG and IG models. These 12 nodes $v_{i}, i=1,2, \cdots, 12$ are equally distributed on the circle centered at $u$ with radius $2 r_{u}$. The interference radius of each of them is set as $r_{u}+\epsilon$ for a very small positive $\epsilon$. Both the transmission radius and the interference radius of $u$ is set as $r_{u}$. A node $w_{i}, i=1,2, \cdots, 12$, is placed on segment $u v_{i}$ and $\left\|u w_{i}\right\|=r_{u}$. Thus, node $u$ has interference with all 12 nodes $v_{i}, i=1,2, \cdots, 12$ in both DG and IG, and these 12 nodes are independent with each other in these two graph models also. It then implies that the lower bound on the approximation ratio for MIS of recursively picking the smallest radius is at least 12 for interference graph model and the disk graph model. We conjecture that 12 is also the tight upper bound, but to prove that, a more rigorous analysis is needed. We leave it as a possible future work.

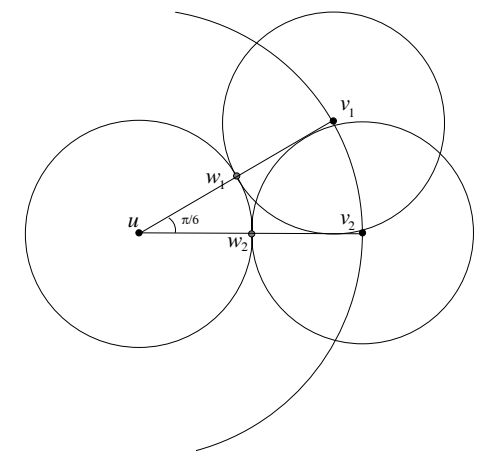

Fig. 4. Twelve independent neighbors are removed by $u$ in DG and IG models.

This simple approximation method for MIS can be easily converted to a distributed one that is suitable for wireless ad hoc networks. It is omitted here due to space limit.

\subsection{PTAS for Maximum Weighted Independent Set}

In this subsection, we will present a PTAS for the maximum weighted independent set (MWIS) for graph models introduced in this paper. Assume that we are given a set $\mathcal{D}=\left\{D_{1}, D_{2}, \cdots, D_{n}\right\}$ of $n$ interference disks in a twodimensional plane, where disk $D_{i}$ has interference radius $r_{i}$, center $v_{i}=\left(x_{i}, y_{i}\right)$ and a weight $w\left(D_{i}\right)=w_{i}$. The weight is typically assigned to node, but here 
we assume that it is assigned to the interference disk $D_{i}$ for later convenience. The transmission radius of node $v_{i}$ is $t_{i}$, which will only be used to determine if two nodes are connected in the corresponding graph model. For a subset of disks $U \subseteq \mathcal{D}$, let $w(U)=\sum_{D_{i} \in U} w\left(D_{i}\right)$, i.e., the summation of the weights of disks in $U$. Two disks $D_{i}$ and $D_{j}$ are said to be independent if the two nodes $v_{i}$ and $v_{j}$ are not connected in the corresponding graph model; otherwise they are called non-independent. Figure 5 shows the non-independence in different graph models.

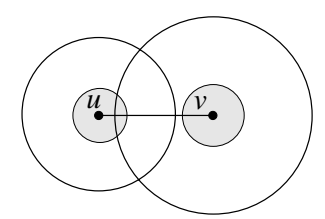

DG

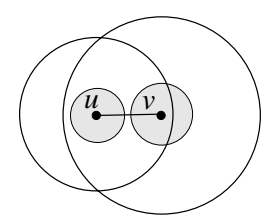

MG

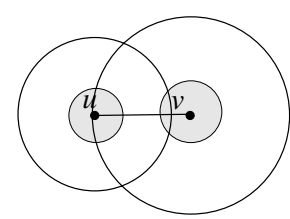

CG

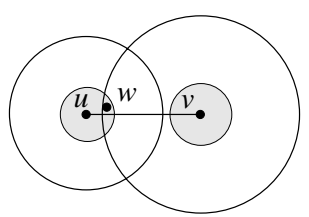

IG

Fig. 5. Non-independence in different graph models. The shaded disks represent the transmission regions and the non-shaded disks represent the interference regions.

Similarly, we also adopt the shifting strategy [30] to develop a PTAS for the maximum weighted independent set problem under various new graph models introduced in this paper. Before presenting our method, let's briefly review the shifting strategy [30] used to develop a PTAS for MIS in the unit disk graph. Here we assume that the radius of each disk is $\frac{1}{2}$. The plane is assumed to be subdivided into grid of size at $m$ by $m$ for some integer $m$ by a collection of vertical lines $x=i \cdot m$ and horizontal lines $y=j \cdot m$. A subdivision is called $(r, s)$-shifting if it is formed by a collection of vertical lines $x=i \cdot m+r$ and horizontal lines $y=j \cdot m+s$, where $0 \leq r, s<m-1$. A square is formed by two consecutive vertical lines and two consecutive horizontal lines in a $(r, s)$ shifting. For each square, an optimal solution of MIS is obtained in polynomial time for all disks contained in the square but not intersecting the boundary of the square. The union of the MIS in all squares is returned as the final solution for this shifting, which clearly is an independent set. Through the pigeonhole principal, it was showed that there is a shifting of the subdivision such that the size of the computed independent set is at least $\left(1-\frac{1}{m}\right)^{2}$ of optimum.

We build our PTAS for approximating MIS in various graph models introduced in this paper based on the approach of [13], i.e., to divide the interference disks into different levels according to their radii. At same level, all nodes have similar interference radii, i.e., they are within a constant factor of each other. As in $[13,29]$, we scale all disks so that the largest disk has interference radius $\frac{1}{2}$. Let $r_{\text {min }}$ be the smallest interference radius among all wireless nodes. Let $k>1$ be a fixed integer (whose value will be specified later) and $\ell=$ $\left\lfloor\log _{k+1} \frac{1}{2 r_{\text {min }}}\right\rfloor$. We partition the set of interference disks $\mathcal{D}$ into $\ell+1$ levels such that level $j, 0 \leq j \leq \ell$, consists of all disks $D_{i}$ with interference radius satisfying that $\frac{1}{(k+1)^{j+1}}<2 r_{i} \leq \frac{1}{(k+1)^{j}}$. Let $l\left(D_{i}\right)$ denote the level of disk $D_{i}$, 
i.e., $l\left(D_{i}\right)=\left\lfloor\log _{k+1} \frac{1}{2 r_{i}}\right\rfloor$. Notice that we do not partition the transmission disks at all. Surprisingly, the solely partition of interference disks is enough to get a PTAS for us.

Similar to the approaches $[13,29,30]$, for each level $j$, we subdivide the plane into grid by using a set of vertical lines $L_{j, v}: x=v \frac{1}{(k+1)^{j}}, v \in Z$ and a set of horizontal lines $H_{j, h}: y=h \frac{1}{(k+1)^{j}}, h \in Z$. Hereafter $j$ is called the level of the lines $L_{j, v}$ and $H_{j, h} ; v$ (and $h$ ) is called the index of the vertical (and horizontal) line $l_{j, v}$ (and $h_{j, h}$ ) at level $j$. A $(r, s)$-shifting of the subdivision is the grid defined by the set of vertical lines whose indices modulo $k$ equal $r$ and the set of horizontal lines whose indices modulo $k$ equal $s$. It was proved in [13] that a vertical line at level $j$ of a $(r, s)$-shifting subdivision is also a vertical line at level $j+1$ of the $(r, s)$-shifting subdivision.

Any two consecutive vertical lines at level $j$ whose indices modulo $k$ equal $r$, and any two consecutive horizontal lines at level $j$ whose indices modulo $k$ equal $s$, form a $j$-square in the $(r, s)$-shifting subdivision. See Figure 6 for an illustration of a 0 -square for $r=s=0$ and $k=3$. In the figure, the solid lines are lines at level 0 and all dashed lines are lines at level 1 . The $j$-squares are represented by thicker lines. The square represented by thicker solid lines is a 0 -square and the squares represented by thicker dashed lines are 1-squares.

Clearly, any $j$-square $S$ is subdivided into $(k+1)^{2}(j+1)$-squares (by lines $L_{j+1, v}$ and $H_{j+1, h}$ at level $\left.j+1\right)$. Notice that it only contains $k^{2}$ grids defined by lines at level $j$. These $(j+1)$-squares $S^{\prime}$ are called the children of $S$, denoted by $S^{\prime} \prec S$. And $S$ is called the parent of $S^{\prime}$. Obviously, any $j$-square $S$ has length $\frac{k}{(k+1)^{j}}$. Notice that an interference disk at level $j$ has radius $r$ satisfying $\frac{1}{(k+1)^{j+1}}<2 r \leq \frac{1}{(k+1)^{j}}$. Thus, a $j$-square can contain some disks inside with level at least $j-1$, but not any disks inside with level less than $j-1$.

A disk $D_{i}$ with center $\left(x_{i}, y_{i}\right)$ and radius $r_{i}$ is said to hit a vertical line at $x=a$ if $a-r_{i}<x_{i} \leq a+r_{i}$. Similarly, we say the disk $D_{i}$ hits a horizontal line at $y=b$ if $b-r_{i}<y_{i} \leq b+r_{i}$. In other words, a disk hits a line if it intersects this line or it touches the line from the left or from the bottom.

For maximum weighted independent set problem, an interference disk $D_{i}$ at level $l\left(D_{i}\right)=j$ is said to be active (respecting to $(r, s)$-shifting) if it does not intersect the boundary of any $j$-square of the $(r, s)$-shifting subdivision. However, the definition of active disks for minimum vertex cover is different, which will be discussed in detail later. Let $\mathcal{D}_{S}$ be the set of disks in $\mathcal{D}$ that are active for $S$. For a $j$-square $S$, let $\mathcal{D}_{S}^{<j}$ be the set of active disks with level less than $j$ and intersecting with $S$. Similarly, we define $\mathcal{D}_{S}^{\leq j}, \mathcal{D}_{S}^{=j}, \mathcal{D}_{S}^{>j}$, and $\mathcal{D}_{\bar{S}}^{\geq j}$ for active disks intersecting $S$ and with level no more than $j$, equal to $j$, larger than $j$, and no less than $j$ respectively. For a $j$-square $S$, let $O P T_{S}^{<j}$ be the set of disks from $O P T\left(\mathcal{D}_{S}\right)$ with level less than $j$ and intersecting with $S$. 
Similarly, we define $O P T_{\bar{S}}^{\leq j}, O P T_{S}^{=j}, O P T_{S}^{>j}$, and $O P T_{\bar{S}}^{\geq j}$ respectively.

For each level $j$, let $\mathcal{D}_{j}(r, s)$ be the set of active interference disks at level $j$ respecting to $(r, s)$-shifting. Define $\mathcal{D}(r, s)=\cup_{j=0}^{\ell} \mathcal{D}_{j}(r, s)$, i.e., the union of active interference disks at all levels respecting to $(r, s)$-shifting. Then a $j$ square $S$ is called relevant if $\mathcal{D}(r, s)$ contains at least one disk of level $j$ that is inside $S$ in the corresponding graph model. More rigorous definition of relevant will be given later, which depends on the graph models. Let $O P T_{I S}\left(\mathcal{D}^{\prime}, G\right)$ denote the weight of the maximum weighted independent set for a set of disks $\mathcal{D}^{\prime}$ when the network is modeled by graph model $G$. We will omit $G$ and/or $I S$ when it is clear from the context. The following proof is given by Erlebach et al. [13] for the disk graph model and $r_{u}=t_{u}$ for each node $u$. We found that the correctness of this lemma does not depend on the graph model. We include it here for the completeness of presentation.

Lemma 3 Given a graph $G$, which is a disk graph, or an interference graph, or a mutual-inclusion graph, or a conflict graph, there is at least one $(r, s)$ shifting, $0 \leq r, s<k$ such that

$$
\operatorname{OPT}(\mathcal{D}(r, s), G) \geq\left(1-\frac{1}{k}\right)^{2} \cdot \operatorname{OPT}(\mathcal{D}, G)
$$

Proof. Consider a maximum weighted independent set $S^{\star} \subseteq \mathcal{D}$ for any graph model introduced here. Let $S_{r}^{\star}$ be the set of disks $D_{i} \in S^{\star}$ such that the disk $D_{i}$ hits some vertical line $L_{j, v}$ at the level $j=l\left(D_{i}\right)$ whose index $v$ modulo $k$ equals $r$. Then $\cup_{r=0}^{k-1} S_{r}^{\star} \subseteq S^{\star}$. In addition, $S_{r}^{\star}, 0 \leq r \leq k-1$ are pairwisely disjoint. Thus, $\sum_{r=0}^{k-1} w\left(S_{r}^{\star}\right) \leq w\left(S^{\star}\right)$. The pigeonhole principal implies that there is an index $r_{0}$ such that $w\left(S_{r_{0}}^{\star}\right) \leq \frac{1}{k} \cdot w\left(S^{\star}\right)$. Let $S_{r_{0}}^{\star}=S^{\star}-S_{r_{0}}^{\star}$. Therefore, $w\left(S_{\overline{r_{0}}}^{\star}\right) \geq\left(1-\frac{1}{k}\right) \cdot w\left(S^{\star}\right)$.

Using the same technique, we can show that there is an index $s_{0}$ such that the set of disks from $S_{\bar{r}_{0}}^{\star}$, among which each $D_{i}$ does not hit a horizontal line $H_{j, h}$ at its level $j=l\left(D_{i}\right)$ and the index $h$ modulo $k$ equals $s_{0}$, has total weight at least $\left(1-\frac{1}{k}\right) \cdot w\left(S_{\overline{r_{0}}}^{\star}\right)$. Use $S_{\bar{r}_{0}, \overline{s_{0}}}^{\star}$ to denote such set of disks, i.e., $S_{\overline{r_{0}}, \bar{s}_{0}}^{\star}=S_{\overline{r_{0}}}^{\star}-S_{\bar{r}_{0}, s_{0}}^{\star}$. Here $S_{\overline{r_{0}}, s_{0}}^{\star}$ is defined similarly to $S_{r}^{\star}$ by replacing $S^{\star}$ with $S_{\overline{r_{0}}}^{\star}$.

Obviously, $S_{\bar{r}_{0}, \overline{s_{0}}}^{\star}$ is an independent set for $\mathcal{D}\left(r_{0}, s_{0}\right)$. Thus, there is a $\left(r_{0}, s_{0}\right)$ shifting such that the weight of the maximum weighted independent set in $\mathcal{D}\left(r_{0}, s_{0}\right)$ is at least $\left(1-\frac{1}{k}\right)^{2}$ of the optimum $O P T(\mathcal{D})$.

The lemma implies the following corollary:

Corollary 4 If we can solve the maximum weighted independent set for disks inside each relevant $j$-square optimally, then we have a PTAS for MWIS, i.e., setting $k=\frac{1+\epsilon+\sqrt{1+\epsilon}}{\epsilon}$ implies that $O P T(\mathcal{D}(r, s), G) \geq \frac{1}{1+\epsilon} \cdot O P T(\mathcal{D}, G)$. 
Before we show the PTASs for MWIS for graph models introduced in this paper, we first exam the structural properties of an optimum solution for all disks in $\mathcal{D}(r, s)$ for $0 \leq r, s \leq k-1$. Hereafter, for convenience, we say two interference disks are independent if the corresponding two nodes are not connected in the corresponding graph model.

Given a graph model, an optimum solution cannot contain any disk that hits a line at level 0 of the $(r, s)$-shifting subdivision. In other words, each disk of the optimum solution $\operatorname{OPT}(\mathcal{D}(r, s))$ is contained inside some 0 -square. Moreover, the optimum solution can be divided into two subsets. One contains some independent interference disks at level 0 , denoted by $I_{0}$. The other one contains independent interference disks at lower level that are independent with any interference disk from $I_{0}$. By the definition of $\mathcal{D}(r, s)$, all interference disks in the second subset cannot intersect any lines, with level 1, of the $(r, s)$-shifting subdivision. In other words, each interference disk in the second subset is contained inside some 1-square. Figure 6 gives an example of optimum solution in a 0 -square. Here $k=3$ and $r=s=0$. The interference disks with the thickest boundary are at level 0 . The interference disks that are not active is represented by dashed boundary. The shaded interference disks are in the optimum solution.

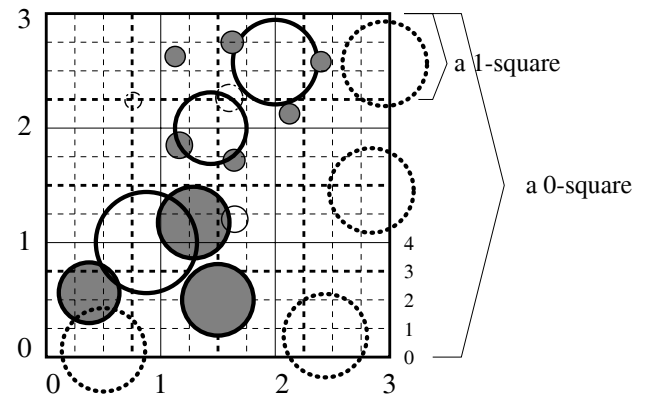

Fig. 6. An optimum solution for a 0 -square when disk graph model is used and $t_{v}=r_{v}$ for each node $v$.

The above partition of interference disks in the optimum solution in a 0-square can be performed recursively down to the squares at level $\ell$ as follows. Given a $j$-square $S$, let $I$ be a set of independent interference disks of level smaller than $j$, each of which intersects $S$. Let $\operatorname{MWIS}(S, I)$ be a maximum weighted independent set of interference disks that are contained in $S$ (must be of level at least $j$ ) and independent from the interference disks in $I$. Then the union of $M W I S(S, \emptyset)$ for all relevant squares $S$ without parent must be the optimum solution for $\mathcal{D}(r, s)$.

We then discuss in detail how to compute $\operatorname{MWIS}(S, I)$ using the dynamic programming. Assume that we already computed the entry $\operatorname{MWIS}(S, I)$ for all squares $S$ with level at least $j+1$ and all appropriate independent sets $I$ intersecting $S$. The interference disks in $\operatorname{MWIS}(S, I)$ can be divided into two subsets. One, denoted by $X$, contains some independent interference disks 
inside $S$ with level $j$ that are independent with interference disks from $I$. The other one contains independent interference disks with level larger than $j$ that are independent with any interference disk from $I$ and $X$. By the definition of $\mathcal{D}(r, s)$, all interference disks in the second subset cannot intersect any lines, with level $j+1$, of the $(r, s)$-shifting subdivision because we only consider active interference disks. In other words, each interference disk in the second subset is contained inside some $(j+1)$-square $S^{\prime}$, which is contained in $S$. Thus, by properly choosing the set of interference disks $X$ (interference disks inside $S$ with level $j$ and independent with $I$ ), we compute $M W I S(S, I)$ from

$$
M W I S(S, I)=\max _{X}\left(\left(\bigcup_{S^{\prime} \prec S} \operatorname{MWIS}\left(S^{\prime}, I_{S^{\prime}} \cup X_{S^{\prime}}\right)\right) \bigcup X\right) .
$$

Here $I_{S^{\prime}}$ is the subset of interference disks from $I$ that intersect $S^{\prime} . X_{S^{\prime}}$ is defined similarly.

The algorithm processes all relevant squares in order of non-increasing levels. For each $j$-square $S$ and some appropriate independent set $I, M W I S(S, I)$ is computed by dynamic programming, as shown in Algorithm 2.

As did in [13], we can easily show that the running time of this algorithm is $O\left(k^{2} n^{C}\right)$. Here $C$ is the constant in Lemma 5. Notice that, recently Chan [31] present a PTAS for maximum weighted independent set for disk graphs defined in [13] with time complexity $n^{O(1 / \epsilon)}$ for two dimensional fat objects. It remains a future work if it also produces PTASs for the MWIS in the graph models introduced in this paper.

It is not difficult to prove the correctness of the above dynamic programming approach. To guarantee that it runs in polynomial time of the number of interference disks $n$ and $k$, we have to show that the size of $I_{S} \cup X_{S}$, i.e., the number of independent interference disks with level at most $j$ and intersecting a $j$-square $S$, is always bounded by a constant under the graph models introduced in this paper.

Lemma 5 Let $S$ be any $j$-square and let I be a set of independent interference disks with level at most $j$, each of which may connect to some interference disks contained in $S$. Then there is a constant $C$ depending on the graph model and $k$ such that the cardinality of $I$ is at most $C$.

Proof. We prove this lemma individually for each graph model.

For mutual-inclusion graph model, the interference disk whose center is outside of $S$ cannot connect with any interference disk contained inside $S$ regardless of the size of their transmission radii. So if we add them to $M W I S(S, I)$, the resulted set is still guaranteed to be an independent set of interference disks. Thus, to bound $|I|$, we only have to consider the interference disks whose 


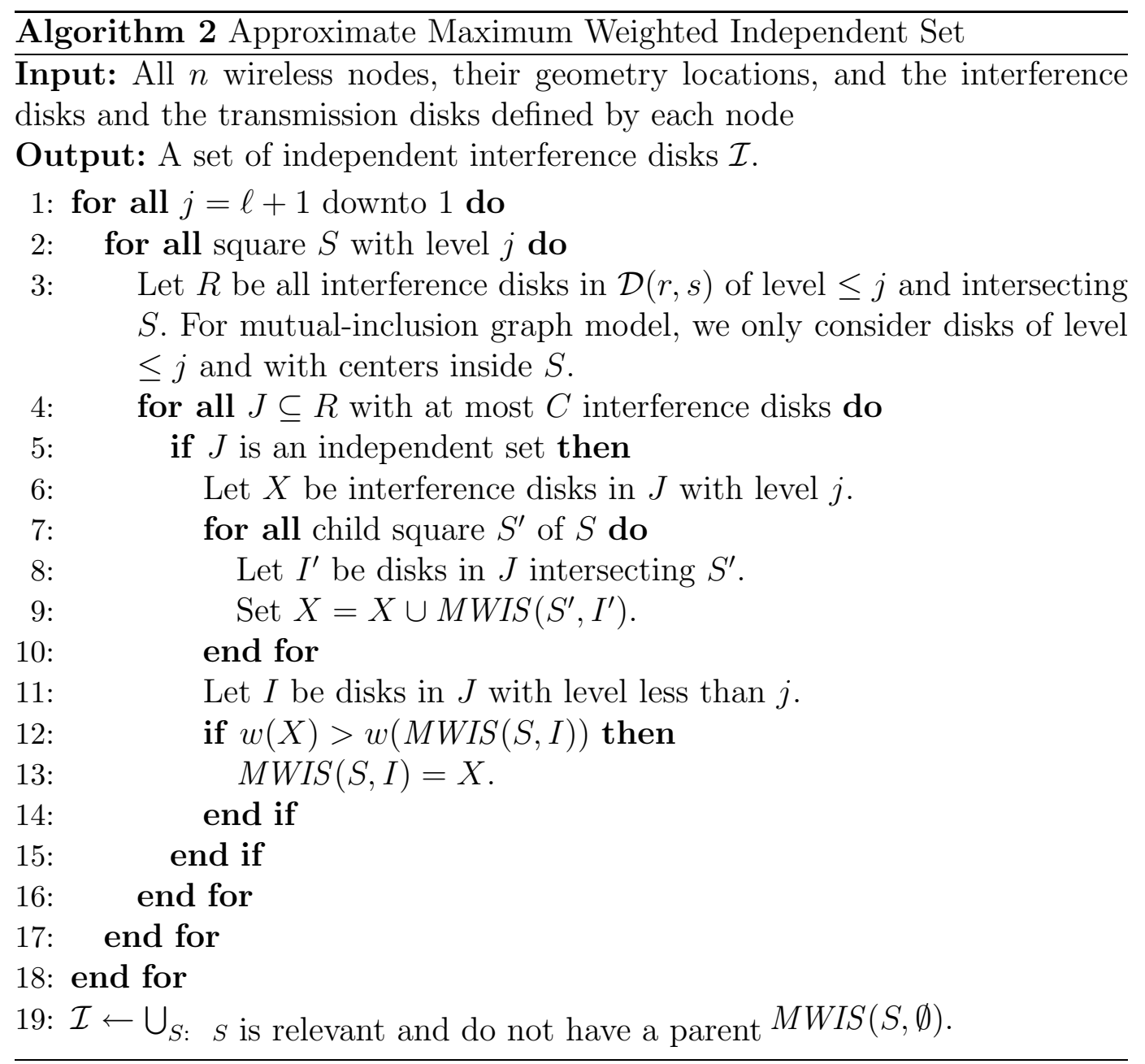

centers are inside $S$, i.e., the interference disks that can possibly "connect" with some interference disk contained inside $S$. Remember that, in the mutualinclusion graph model, we say two interference disks centered at nodes $u$ and $v$ are connected iff $u$ and $v$ are inside $I(u, v)$. Notice that all interference disks in $I$ have level at most $j$, which implies that each interference disk in $I$ has a diameter at least $\frac{1}{(k+1)^{j+1}}$. Then the distance between the centers of any two interference disks $D_{p}$ and $D_{q}$ from $I$ is at least $\frac{1}{2(k+1)^{j+1}}$; otherwise, these two disks $D_{p}$ and $D_{q}$ will not be independent in $\mathrm{MG}$. The $j$-square $S$ has side length $\frac{k}{(k+1)^{j}}$. Therefore, there are at most a constant $C_{M G}$ independent disks whose centers are inside $S$. See Figure 7 . Here, by an area argument,

$$
C_{M G} \leq\left(\frac{k}{(k+1)^{j}}\right)^{2} /\left(\pi\left(\frac{1}{4(k+1)^{j+1}}\right)^{2}\right)=\frac{16 k^{2}(k+1)^{2}}{\pi} .
$$

For conflict graph model, again, we only have to consider all independent interference disks that could connect to some interference disks contained in $S$. Remember that, here, two interference disks $D_{p}$ and $D_{q}$ are independent if $v_{p}$ and $v_{q}$ are not inside $I\left(v_{p}, v_{q}\right)$. Equivalently, $v_{p}$ is not inside $D_{q}$ and $v_{q}$ is 

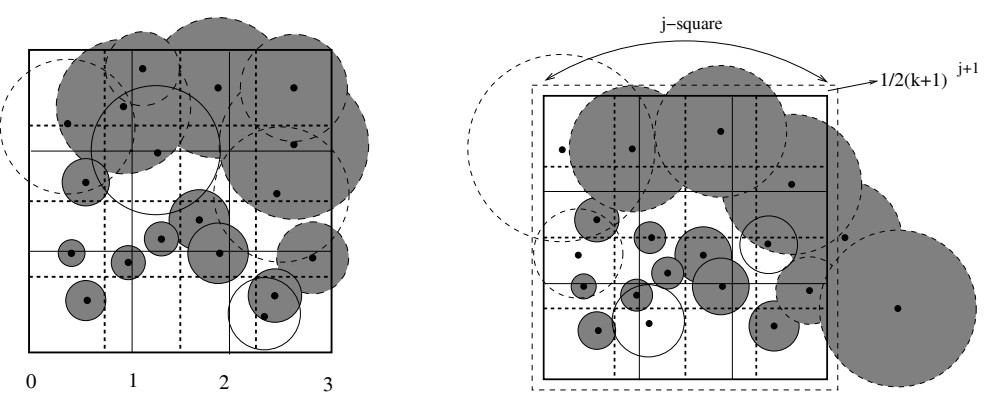

Fig. 7. There are at most a constant number of independent interference disks of level at most $j$, intersecting a $j$-square $S$ in MG (left) and CG (right). Only the interference disks are drawn. The transmission disks are omitted. Here $k=3$ and the largest square is $j$-square. The smaller dashed squares are $(j+1)$-squares.

not inside $D_{p}$. Thus, the distance between the centers of any two disks from $I$ is at least $\frac{1}{2(k+1)^{j+1}}$, i.e., the radius of the possible smallest interference disks of level $j$. Using the same area argument as for MG, we can show that there are at most $\frac{16 k^{2}(k+1)^{2}}{\pi}$ independent interference disks whose centers are inside $S$. Then we concentrate on estimating how many independent interference disks, denoted by $I_{O}$, such that (1) their centers are not inside $S$, (2) with level at most $j,(3)$ each of them intersects $S$. We show that there are only a constant number of such interference disks by an area argument. Consider the four strips, denoted by $B(S)$, surrounding $S$ with width $\frac{1}{2(k+1)^{j+1}}$. See Figure 7 for an illustration. For an interference disk $D_{i} \in I_{O}$, it is not difficult to show that $B(S) \cap D_{i}$ achieves the smallest area when $v_{i}$ is on the boundary of $B(S)$ and $r_{v_{i}}=\frac{1}{2(k+1)^{j+1}}$. The smallest area of $B(S) \cap D_{i}$ is $\pi \frac{1}{8(k+1)^{2(j+1)}}$. Similar to Theorem 2, every point in $B(S)$ is covered by at most 5 interference disks from $I_{O}$. The area of $B(S)$ is $\frac{2 k(k+1)+1}{(k+1)^{2(j+1)}}$. Thus, the size of $I_{O}$ is at most

$$
5 \cdot \frac{2 k(k+1)+1}{(k+1)^{2(j+1)}} /\left(\pi \frac{1}{8(k+1)^{2(j+1)}}\right)=\frac{80 k(k+1)+40}{\pi}
$$

Thus, the total number of independent interference disks $I$ with level at most $j$ and intersecting $S$ is at most

$$
C_{C G} \leq \frac{16 k^{2}(k+1)^{2}}{\pi}+\frac{80 k(k+1)+40}{\pi} .
$$

For interference graphs, as we already showed that $C G \subseteq I G$, any independent set in $I G$ is also an independent set in $C G$. For the conflict graphs, we considered all interference disks that intersect the $j$-square and for interference graph model, we also have to consider all interference disks intersecting the $j$-square. Thus, $C_{C G}$ is also an upper-bound of the number of independent disks with level at most $j$ intersecting $S$ for interference graph model.

For disk graph model, there are two approaches to bound the number of in- 
dependent interference disks with level at most $j$ that intersect the $j$-square, denoted by $C_{D G}$. One approach is to use the fact that $C G \subseteq D G$ and the set of interference disks from which to select an independent set is same for disk graph model and the conflict graph model. Thus, we have $C_{D G} \leq C_{C G}$. The other approach is to follow the analysis for conflict graph model. Since if two interference disks are independent then the distance between their centers is at least the smaller radius of these two disks. Following the analysis for CG, we will get the exact same bound $C_{D G}$ for the disk graph model.

This finishes the proof of the lemma.

Remark 1: The main contributions of our PTAS compared with the method presented in [13] are the definition of active disk respecting to a $(r, s)$-shifting, and the new method (stated in Lemma 5) to include which disks should be considered when processing a square $S$. Notice that the simple extension of method in [13] will not work here because the number of independent interference disks that intersect a $j$-square in the mutual-inclusion graph model is not bounded by any constant. It is easy to show that the disks $D_{i}, i \geq 1$, with center $v_{i}=\left(2^{i-1}(1+\epsilon)^{i}, 0\right)$ and radius $r_{i}=2^{i-1}(1+\epsilon)^{i}$ are independent; all such disks intersect any square containing the point $(0,0)$. Here $\epsilon$ is a small positive real number.

Remark 2: The other contribution is that although the underlying graph models require the transmission radius of each node (thus, the independence of nodes requires the nodes' transmission radii), we introduced a new concept of independence among the interference disks only. Consequently, in our analysis of the upper bound of the number of independent interference disks that intersect a $j$-square, the transmission radius $t_{v}$ of every node $v$ does not play any role here. Notice that we do not require any relations among the transmission radii and the interference radii of all nodes, except that the interference radius of each node is at least its transmission radius.

Remark 3: Our PTAS can be extended further as long as Lemma 5 holds even the transmission regions and the interference regions are not disks.

\section{Vertex Cover}

The second problem we study is vertex cover problem which also has been studied and applied in wireless networks [48-50]. It is well-known that the minimum weighted vertex cover for a general graph can be approximated within 2. In this section, we present a PTAS for the minimum weighted vertex cover problem for all graph models introduced here. As we did for approximating MWIS, we use the same partition of interference disks into levels and 
the same subdivision of the plane into squares at each level. In addition, all integer values $r$ and $s$ within interval $[0, k-1]$ are considered. We first study the structural properties of the MWVC. Let $O P T$ be the minimum weighted vertex cover for $\mathcal{D}$. Let $O P T V(r)$ be the set of interference disks $D_{i} \in O P T$ such that the interference disk $D_{i}$ hits some vertical line $L_{j, v}$ at the level $j=l\left(D_{i}\right)$ whose index $v$ modulo $k$ equals $r$. Similarly, let $O P T H(s)$ be the set of interference disks $D_{i} \in O P T$ such that the interference disk $D_{i}$ hits some horizontal line $H_{j, h}$ at the level $j=l\left(D_{i}\right)$ whose index $h$ modulo $k$ equals $s$. Let $O P T(r, s)=O P T V(r) \cup O P T H(s)$.

Lemma 6 There is a $\left(r_{0}, s_{0}\right)$-shifting such that the total weight of interference disks in OPT $\left(r_{0}, s_{0}\right)$ is no more than $\frac{2}{k}$ of the weight of OPT.

Proof. Obviously, OPTV $(r)$ are pair-wisely disjoint for $0 \leq r<k$ and $\sum_{r=0}^{k-1} w(O P T V(r))=w(O P T)$. Through pigeonhole principal, there is an integer $r_{0}$ such that $w\left(O P T V\left(r_{0}\right)\right) \leq \frac{1}{k} w(O P T)$. Similarly, there exists $s_{0}$ such that $w\left(O P T H\left(s_{0}\right)\right) \leq \frac{1}{k} w(O P T)$. Therefore, $w\left(O P T\left(r_{0}, s_{0}\right)\right) \leq w\left(O P T V\left(r_{0}\right)\right)+$ $w\left(O P T H\left(s_{0}\right)\right) \leq \frac{2}{k} w(O P T)$.

The idea of this proof is given by Erlebach et al. [13] for the disk graph model and $r_{u}=t_{u}$ for each node $u$. We found that the correctness of this lemma does not depend on the graph model. We include it here for the completeness of presentation.

For independent set problem, to guarantee that the union of independent set of interference disks in each child of a square $S$ is still an independent set, we only consider the independent disks that are totally contained in the children of $S$. In other words, an interference disk with level $j$ is active for MWIS if it is geometrically inside some $j$-square. Contrary to MWIS, a vertex cover of nodes whose interference disks are inside a square could use some nodes whose interference disks are not totally inside in this square. Then we have to define active disks for a $j$-square differently from MWIS. As the vertex cover has to cover all edges, we associate each edge to at least one square and then compute the vertex cover for all edges associated with that $j$-square.

For graph model DG, IG, and CG, we associate an edge $u v$ to a $j$-square $S$ if $S$ intersects with $D_{u} \cap D_{v}$. Obviously, in any graph model introduced in this paper, if $u v$ is an edge then the interference disk $D_{u}$ intersects the interference disk $D_{v}$. In addition, there is always some square $S$ that has nonempty intersection with $D_{u} \cap D_{v}$. Thus, any edge $u v$ is associated with some square. See the left figure in Figure 8 for an illustration.

For graph model MG, we associate the edge $u v$ to a $j$-square $S$ if $S$ intersects with $D_{u} \cap D_{v}$ and $S$ contains $u$ inside and either (1) $r_{u}<r_{v}$ or $(2) r_{u}=r_{v}$ and the identity $I D(u)$ of $u$ is less than $I D(v)$. For any edge $u v$, assume that $u \in S_{1}$ and $r_{u} \leq r_{v}$. Then $S_{1} \cap\left(D_{u} \cap D_{v}\right) \neq \emptyset$ because $u \in D_{u} \cap D_{v}$. So edge 
$u v$ must be associated with square $S_{1}$. See the right figure in Figure 8.

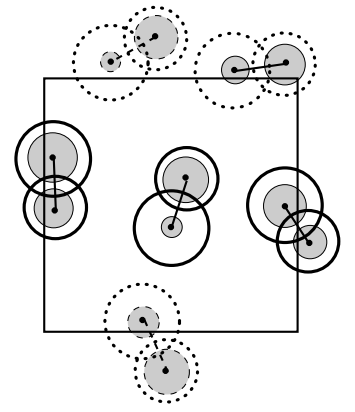

a) non-mutual-inclusion

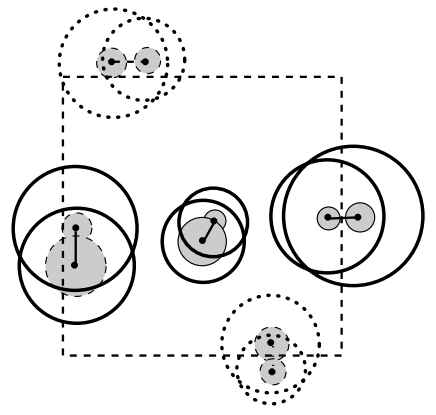

(b) mutual-inclusion

Fig. 8. Examples for the associated edges and the active disks.

For a $j$-square $S$, an interference disk $D_{u}$ is said to be active for $S$ if there is an edge $u v$ associated with $S$ for some $v$. Thus, for edges associated with a $j$-square $S$, their vertex cover must be a subset of the active interference disks in $S$. Notice that an active interference disk $D_{u}$ for a $j$-square $S$ always intersects $S$ in any graph model. See Figure 8 as an illustration. Here the dashed interference disks are inactive, the solid interference disks are active for $S$. For all graph models, each interference disk is at most active for four $j$-squares on the same level of this disk.

A $j$-square $S$ is called relevant if there are some edges in the corresponding graph model associated with $S$. Given a $j$-square $S$, the presented algorithm will then construct a vertex cover for the edges associated with $S$ using interference disks active for $S$ with a prior assumption that some active interference disks $P$ are already chosen to be in the vertex cover. More precisely, given some subset $P$ of active interference disks for a $j$-square $S$, we will compute the minimum weighted set of active interference disks $X$, denoted by $V C(S, P)$, such that $P \cup X$ is a vertex cover for edges associated with $S$.

For each $j$-square $S$, we consider all the interference disks each of which is incident on some edges associated with $S$. We then consider the optimum solution $O P T$ restricted to a square $S$, denoted by $O P T_{S}$, i.e., the interference disks in $O P T$ that are active for $S$. Then, $O P T_{S}$ is a vertex cover for all edges associated with $S$. Define active edges of $S$ as the set of edges $u v$ with interference disks $D_{u}$ and $D_{v}$ are active for $S$. Then the active edges of $S$ contains the associated edges of $S$. Thus, $O P T_{S}$ is also a vertex cover for all active edges of $S$.

Consider a relevant 0 -square $S$, the optimum solution $O P T_{S}$ can be divided into two subsets. One contains the interference disks which is a complement of an independent set of active interference disks for $S$ at level 0 , denoted by $P_{0}$. The other one contains disks at lower level that, together with $P_{0}$, can form a vertex cover for all edges active for $S$. Figure 9 gives an example of optimum 
solution restricted in a 0 -square. Here $k=3$ and $r=s=0$. The interference disks with the thickest boundary are at level 0 . The interference disks that are not active are represented by dashed boundary. The un-shaded disks are in the optimum solution. The shaded disks are an independent set.

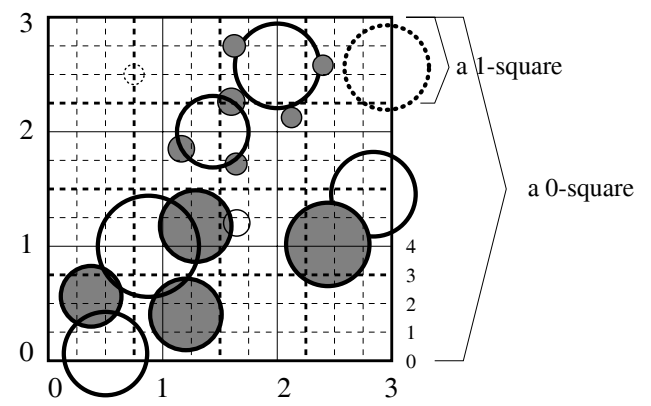

Fig. 9. Active disks and a vertex cover (shaded disks) for a 0-square in disk graph model. Here the transmission disks are not drawn for simplicity.

The above partition of interference disks in the optimum solution in a relevant 0 -square can be performed recursively down to the squares at level $\ell$ as follows. Given a $j$-square $S$, let $R$ be the set of all active interference disks for $S$ with level at most $j$. Then any active edge using only interference disks from $R$ must have vertex cover from $R$. Thus, for any vertex cover $U, R-U$ must be an independent set. Hereafter, we will use $J$ to denote a set of independent and active interference disks with level at most $j$ for a $j$-square $S$. Let $Q=R-J$, i.e., the complement set of interference disks of $J$. Then, $Q$ can cover all interference disk in $R$. Here, we say an interference disk $D_{u}$ covers another interference disk $D_{v}$, if there is an edge $u v$ in the corresponding graph model. Let $P=Q^{<j}$. Let $V C(S, P)$ be the set of minimum weighted interference disks such that $V C(S, P) \cup P$ is a vertex cover for all active edges for $S$. Notice that, for optimum solution $O P T$ restricted to $S$, we can set $Q=O P T_{\bar{S}}^{\leq j}$, $P=O P T_{S}^{<j}, J=R-O P T_{S}^{<j}$. The union of $V C(S, \emptyset)$ for all relevant squares $S$ without parent must be a vertex cover for the graph defined on $\mathcal{D}$.

A dynamic programming to compute $V C(S, \emptyset)$ is straightforward. The algorithm presented here is similar to that of [13]. It processes all relevant squares in order of non-increasing levels. For each $j$-square $S$ and some set $P, \operatorname{VC}(S, P)$ is computed by dynamic programming, as shown in Algorithm 3.

For the base situation (without a relevant child), we actually try all the complement sets of possible independent active interference disks to get the optimum solution with minimum weight. The output of the algorithm is the union of the $V C(S, \emptyset)$, taken over all relevant squares $S$ that do not have a parent. Similar to Algorithm 2, the running time of this algorithm is $O\left(k^{2} n^{C_{2}}\right)$. Here $C_{2}$ is a constant that will be proved by the following Lemma 7 .

Lemma 7 Let $S$ be any $j$-square and $J$ be a set of independent interference disks with level at most $j$ that is active for $S$. There is a constant $C_{2}$ depending 


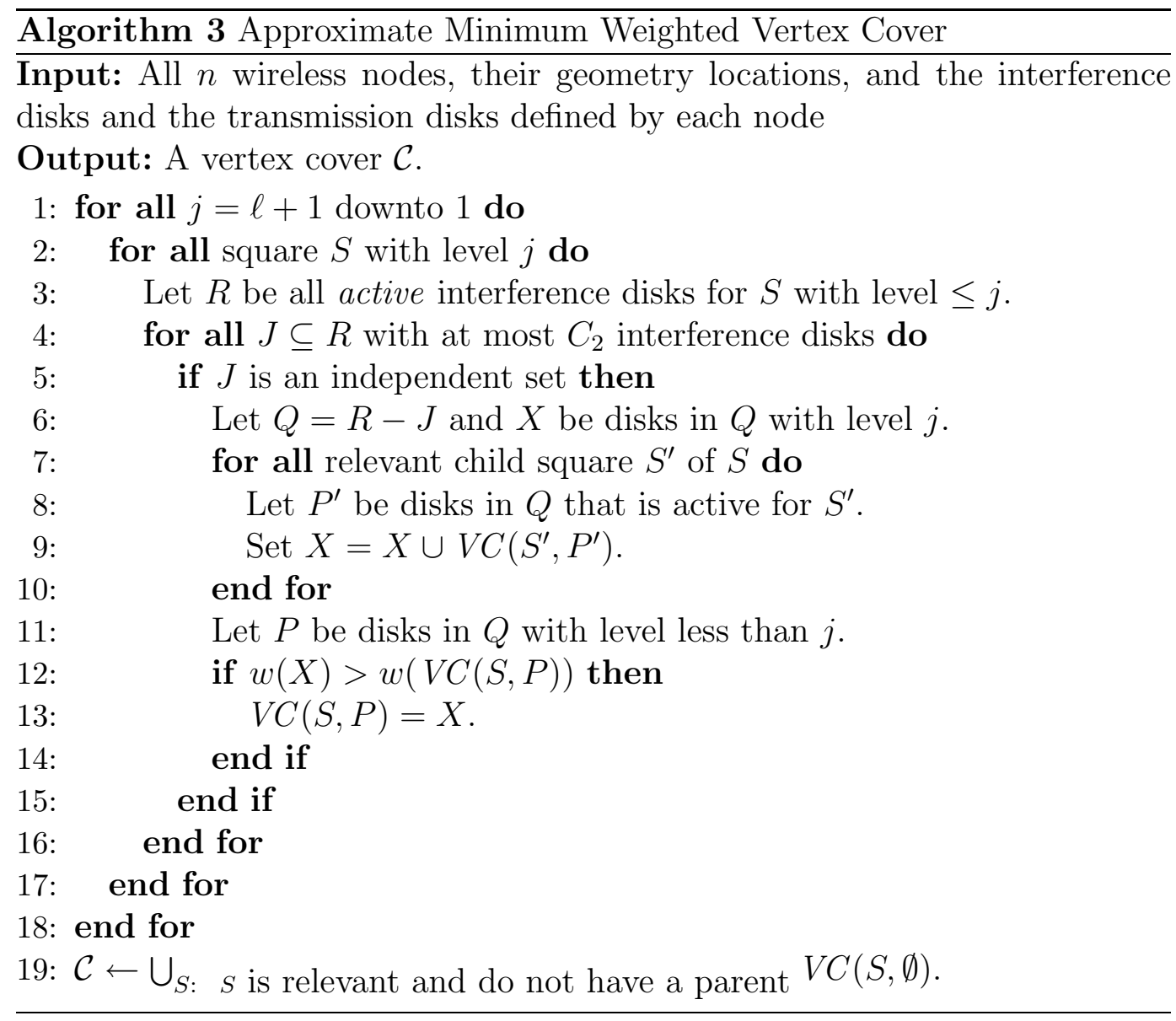

on the graph model and $k$ such that the cardinality of $J$ is at most $C_{2}$.

Proof. For the graph models DG, IG, and CG, all active interference disks intersect the $j$-square $S$. Then, the number of interference disks in $J$ is bounded by a constant using an area argument, which is the same as Lemma 5 .

We then consider the graph model MG. For each interference disk $D_{u} \in J$ with center $u$ outside $S$, there is an interference disk $D_{v}$ with level at least $j$ and $u v$ is an edge in MG. Observe that $u$ must be inside $D_{v}$ regardless of the size of the transmission radii $t_{u}$ and $t_{v}$. Thus, $u$ is always inside the extended square $S \cup B(S)$, where $B(S)$ is same to the definition in the proof of Lemma 5 . Similarly, the number of independent active interference disks is at most

$$
C_{2} \leq\left(\frac{1}{(k+1)^{j-1}}\right)^{2} /\left(\pi\left(\frac{1}{4(k+1)^{j+1}}\right)^{2}\right)=\frac{16(k+1)^{4}}{\pi}
$$

This finishes the proof.

The following lemma shows that our algorithm does compute a valid vertex cover.

Lemma 8 Given $S$ and $P$, our algorithm does compute $X=V C(S, P)$ such 
that $X \cup P$ is a vertex cover for all edges associated with $S$.

Proof. For any edge $u v$ associated to $S$, without loss of generality, we assume that $r_{u} \leq r_{v}$.

If the interference disk $D_{u}$ has level $j$, then both $D_{u}$ and $D_{v}$ are in $R$. Obviously, not both $D_{u}$ and $D_{v}$ can belong to some independent set $J$. In other words, either $D_{u}$ or $D_{v}$ or both is selected to $Q$. If $D_{u}$ is selected to $Q$, then $D_{u}$ is also selected to $X$. So $X \cup P$ covers edge $u v$. If $D_{u}$ is not selected to $Q$, then $D_{v}$ must be selected to $Q$. Then $D_{v}$ will be put into $X$ if $l\left(D_{v}\right)=j$ or it will be put to $P$ if $l\left(D_{v}\right)<j$. Thus, $X \cup P$ covers edge $u v$.

If $D_{u}$ has level larger than $j$, then edge $u v$ is also associated with some child square $S^{\prime}$ of $S$. By induction, $V C\left(S^{\prime}, P^{\prime}\right)$ covers edge $u v$, where $P^{\prime}$ is the disks in $Q$ that are active for $S^{\prime}$. Since $V C(S, P)=X \cup \bigcup_{S^{\prime} \prec S} V C\left(S^{\prime}, P^{\prime}\right)$, we know that $u v$ is also covered by $V C(S, P)$.

Since every edge is active for some square, the output of our algorithm is a vertex cover of $\mathcal{D}$.

We then study the quality of the computed vertex cover. Let $O P T$ be the minimum weighted vertex cover of $\mathcal{D}$ in a graph model introduced in this paper. The following theorem shows that the shifting strategy does work for MWVC problem. The proof of this theorem is omitted here since it is similar to a theory proved in [13] for disk graph model when $t_{v}=r_{v}$ for every node $v$.

Theorem 9 For all graph models introduced here, there is a $(r, s)$-shifting, $0 \leq r, s<k$ such that for the vertex cover $\mathcal{C}$ generated by the algorithm,

$$
w(\mathcal{C}) \leq\left(1+\frac{6}{k}\right) w(O P T) .
$$

Remark: The main contributions of our PTAS compared with the method presented in [13] are the definition of active disk respecting to a $(r, s)$-shifting, the new method to associate edges to a $j$-square, and the proof of Lemma 7.

\section{Graph Coloring}

In this section, we study how to color the graphs introduced in this paper. To begin with, let $\delta(G)$ denote the largest $d$ such that $G$ contains a subgraph $H$ in which each vertex has degree at least $d$. It was proved by Szekeres and Wiff [32] that, every graph $G$ can be colored in $\delta(G)+1$ colors. Then Hochbaum [33] presented a method to find the value of $\delta(G)$ and gave an efficient method 
to color $G$ using $\delta(G)+1$ colors in $O(|V|+|E|)$ time. For the completeness of presentation, we review the algorithm here.

To evaluate $\delta(G)$, it dismantles $G$ by successive removals of vertices of minimum degree and all incident edges. Let $v_{i}$ denote the $i$ th vertex removed from $G$ and $G_{i+1}$ be the graph after $v_{i}$ is removed (set $G_{1}=G$ ). The degree of $v_{i}$ in graph $G_{i}$ is called its valid degree. Set $\delta(G)$ as the maximum valid degree of all nodes. Let $v_{j}$ be the node with the maximum valid degree $\delta(G)$. Then, $v_{j}$ has $\delta(G)$ neighbors among the vertices $v_{j+1}, v_{j+2}, \cdots, v_{n}$. In, addition, all nodes $v_{i}$ with $i>j$ have degree at least $\delta(G)$ in graph $G_{j}$. To color $G$ in no more than $\delta(G)+1$ colors, it scans the sequences of $v_{i}$ 's from $v_{n}$ to $v_{1}$ and assigns to each $v_{i}$ the smallest positive integer not yet assigned to any of its neighbors.

Theorem 10 The above coloring method achieves a constant approximation ratio for graph models $D G, C G, M G, I G$.

Proof. It was already known that, for any graph $G, G$ can be colored by $\delta(G)+1$ colors. Let $H$ be a subgraph such that all nodes have at least $\delta(G)$ degree in $H$. Let $u$ be the node of $H$ with the smallest interference radius. Let $N_{H}(u)$ be all neighbors of $u$ in $H$. Then $\left|N_{H}(u)\right| \geq \delta(G)$. Consider the induced coloring on $N(u)$ by any coloring of $G$. The nodes in $N(u)$ with the same color form an independent set. It was proved in Theorem 1 and Theorem 2 that, if $u$ has an interference radius less than all its neighbors $N_{H}(u)$, then the maximum independent set in $N_{H}(u)$ has size at most 40,5,5 and 40 for the disk graph model, the mutual-inclusion graph model, the conflict graph model, and the interference graph model respectively. Let $O P T$ be the chromatic number of the corresponding graph. There are only $O P T-1$ colors for nodes in $N_{H}(u)$. Then $\left|N_{H}(u)\right| \leq 5(O P T-1)$ for mutual-inclusion graph and the conflict graph, while $\left|N_{H}(u)\right| \leq 40(O P T-1)$ for the disk graph and the interference graph. Thus, the colors used by the above method is at most $5 \cdot O P T-4$ for MG and CG, while at most $40 \cdot O P T-39$ for DG and IG.

\section{Summary and Future Work}

We first proposed several new graph models for wireless ad hoc networks, namely, the disk graphs (DG), the conflict graphs (CG), the mutual-inclusion graphs $(\mathrm{MG})$, the interference graphs (IG), and the mutual communication graphs (MCG) by assuming that each wireless node has an interference disk and a transmission disk. We showed that the maximum independent set problem (MIS) and the minimum graph coloring problem (MGC) can be approximated by simple approximation algorithms within 40, 40, 5, 5 and 5 for DG, IG, CG, MG, and MCG respectively. We also presented PTASs for the 
weighted versions of MIS and the minimum vertex cover problem (MVC) for all graph models. The time complexity of our PTASs is $O\left(\frac{1}{\epsilon^{2}} n^{O\left(\frac{1}{\epsilon^{4}}\right)}\right)$. All of these proposed simple approximation algorithms and PTASs can be used in various applications for wireless ad hoc networks.

One of the most challenging problems is to design a PTAS for dominating set and the connected dominating set problems for introduced graph models, if it is possible. These has been studied in $[30,53]$ for unit disk graph when each node only has one transmission disk. However, little is known for the graph models presented in this paper, even the simpler case when nodes only have transmission disks, and different nodes may have different transmission radii. We leave these problems as future work.

\section{References}

[1] K. Xu, M. Gerla, S. Bae, How effective is the IEEE 802.11 RTS/CTS handshake in ad hoc networks?, in: Proc. of IEEE Global Telecommunications Conference, 2002.

[2] S. Xu, T. Saadawi, Does the IEEE 802.11 MAC protocol work well in multihop wireless ad hoc networks?, IEEE Communications Magazine 39 (6) (2001) 130137

[3] J. Li, C. Blake, D. S.J. De Couto, H. I. Lee, R. Morris, Capacity of ad hoc wireless networks, in: Proceedings of the 7 th annual international conference on Mobile computing and networking, 2001.

[4] T.-C. Tsai, C.-M. Tu, An adaptive IEEE 802.11 MAC in multihop Wireless ad hoc networks considering large interference range, in: Proceedings of Conference on Wireless On-Demand Network Systems (WONS2004), LNCS2928, 2004.

[5] M. C. Golumbic, Algorithmic Graph Theory and Perfect Graphs, Academic Press, New York., 1980.

[6] T. A. McKee, F. R. McMorris, Topics in Intersection Graph Theory, Vol. 2 of Monographs on Discrete Mathematics and Applications, SIAM, 1999.

[7] F. Roberts, Graph Theory and Its Applications to Problems of Society, SIAM Press, Philadelphia, PA., 1978.

[8] J. M. Keil, D. Schaefer, An optimal algorithm for finding dominating cycles in circular-arc graphs, Discrete Applied Mathematics (1992) 25-34.

[9] H. Breu, D. G. Kirkpatrick, Unit disk graph recognition is NP-hard, Computational Geometry. Theory and Applications 9 (1-2) (1998) 3-24.

[10] B. Clark, C. Colbourn, D. Johnson, Unit disk graphs, Discrete Mathematics 86 (1990) 165-177. 
[11] M. V. Marathe, H. Breu, H. B. Hunt III, S. S. Ravi, D. J. Rosenkrantz, Simple heuristics for unit disk graphs, Networks 25 (1995) 59-68.

[12] T. P. William, Three-dimensional geometry and topology, The Geometry Center, University of Minnesota, Minneapolis, MN., 1991.

[13] T. Erlebach, K. Jansen, E. Seidel, Polynomial-time approximation schemes for geometric graphs, in: Proc. of Annual Symposium on Discrete algorithms, 2001.

[14] D. S. Hochbaum, W. Maass, Approximation schemes for covering and packing problems in image processing and vlsi, Journal of ACM 32 (1985) 130-136.

[15] K. M. Alzoubi, P.-J. Wan, O. Frieder, New distributed algorithm for connected dominating set in wireless ad hoc networks, in: Proc. of IEEE HICSS, 2002.

[16] S. Basagni, Distributed clustering for ad hoc networks, in: Proceedings of the IEEE International Symposium on Parallel Architectures, Algorithms, and Networks (I-SPAN), 1999, pp. 310-315.

[17] B. Das, V. Bharghavan, Routing in ad-hoc networks using minimum connected dominating sets, in: Proc. of IEEE International Conference on on Communications (ICC'97), Vol. 1, 1997, pp. 376-380.

[18] J. Wu, H. Li, A dominating-set-based routing scheme in ad hoc wireless networks, the special issue on Wireless Networks in the Telecommunication Systems Journal 3 (2001) 63-84.

[19] I. Stojmenovic, M. Seddigh, J. Zunic, Dominating sets and neighbor elimination based broadcasting algorithms in wireless networks, IEEE Transactions on Parallel and Distributed Systems 13 (1) (2002) 14-25.

[20] Y. Wang, X.-Y. Li, Geometric spanners for wireless ad hoc networks, in: Proc. of 22nd IEEE International Conference on Distributed Computing Systems (ICDCS), 2002.

[21] K. Alzoubi, X.-Y. Li, Y. Wang, P.-J. Wan, O. Frieder, Geometric spanners for wireless ad hoc networks, IEEE Transactions on Parallel and Distributed Processing 14 (4) (2003) 408-421, short version in IEEE ICDCS 2002.

[22] Y. Wang, W. Wang, X.-Y. Li, Efficient distributed low-cost backbone formation for wireless networks, in: ACM International Symposium on Mobile Ad Hoc Networking and Computing (MobiHoc), 2005.

[23] S. Sen, D. F. Towsley, Z.-L. Zhang, J. K. Dey, Optimal multicast smoothing of streaming video over an internetwork, in: Proc. of IEEE INFOCOM, 1999.

[24] H. Tamura, K. Watanabe, M. Sengoku, S. Sengoku, Graph theoretical considerations of a channel assignment problem on multihop wireless networks, in: Proc. of International Technical Conference On Circuits/Systems, Computers and Communications, 2002.

[25] X. Wu, C. Yuen, Y. Gao, H. Wu, B. Li, Fair scheduling with bottleneck consideration in wireless ad-hoc networks, in: Proc. of IEEE International Conference on Computer Communications and Networks (ICCCN 2001), 2001. 
[26] V. S. A. Kumar, M. V. Marathe, S. Parthasarathy, A. Srinivasan, End-to-end packet-scheduling in wireless ad-hoc networks, in: SODA '04: Proceedings of the fifteenth annual ACM-SIAM symposium on Discrete algorithms, 2004.

[27] R. Negi, A. Rajeswaran, Physical layer effect on mac performance in ad-hoc wireless networks, in: Proc. Commun., Internet and Info. Tech. (CIIT 2003), 2003 .

[28] Y.-H. Tseng, E. H. kuang Wuy, G.-H. Chen, Maximum traffic scheduling and capacity analysis for ieee 802.15.3 high data rate mac protocol, in: Proc. of IEEE Semiannual Vehicular Technology Conference (VTC Fall 2003), 2003.

[29] X.-Y. Li, Y. Wang, Simple heuristics and PTASs for intersection graphs in wireless ad hoc networks, in: Proc. of ACM 6th International Workshop on Discrete Algorithms and Methods for Mobile Computing and Communications (DIALM2002), 2002.

[30] I. Harry B. Hunt, M. V. Marathe, V. Radhakrishnan, S. S. Ravi, D. J. Rosenkrantz, R. E. Stearns, NC-approximation schemes for NP- and PSPACEhard problems for geometric graphs, J. Algorithms 26 (2) (1998) 238-274.

[31] T. Chan, Polynomial-time approximation schemes for packing and piercing fat objects, 2001, submitted to Journal of Algorithms.

[32] G. Szekeres, H. Wilf, An inequality for the chromatic number of a graph, Journal of Combinatorial Theory 4 (1968) 1-3.

[33] D. S. Hochbaum, Efficient bounds for the stable set, vertex cover, and set packing problems, Discrete Applied Mathematics 6 (1983) 243-254.

[34] R. Sivakumar, B. Das, V. Bharghavan, The clade vertebrata: spines and routing in ad hoc networks, in: Proc. of IEEE Symposium on Computers and Communications (ISCC98), Athens, Greece, 1998.

[35] J. Wu, F. Dai, M. Gao, I. Stojmenovic, On calculating power-aware connected dominating sets for efficient routing in ad hoc wireless networks, IEEE/KICS Journal of Communication and Networks 4 (1) (2002) 59-70.

[36] B. Liang, Z. J. Haas, Virtual backbone generation and maintenance in ad hoc network mobility management, in: Proc. of Annual Joint Conference of the IEEE Computer and Communications Societies (INFOCOM), 2000.

[37] S. Basagni, Finding a maximal weighted independent set in wireless networks, Telecommunication Systems, Special Issue on Mobile Computing and Wireless Networks 18 (1/3) (2001) 155-168.

[38] M. Chatterjee, S. K. Das, D. Turgut, A weight based distributed clustering algorithm for mobile ad hoc networks, in: Proceedings of Seventh International Conference on High Performance Computing (HiPC), 2000, pp. 511-521.

[39] M. Chatterjee, S. Das, D. Turgut, WCA: A weighted clustering algorithm for mobile ad hoc networks, Journal of Cluster Computing 5 (2) (2002) 193-204. 
[40] C. Bettstetter, R. Krausser, Scenario-based stability anlysis of the distributed mobility-adaptive clustering (DMAC) algorithm, in: Proc. of the 2nd ACM international symposium on mobile ad hoc networking \& computing, 2001.

[41] U. C. Kozat, G. Kondylis, B. Ryu, M. Marina, Virtual dynamic backbone for mobile ad hoc networks, in: Proceedings of IEEE ICC 2001, 2001.

[42] A. D. Amis, R. Prakash, Load-balancing clusters in wireless ad hoc networks, in: Proceedings of the 3rd IEEE Symposium on Application-Specific Systems and Software Engineering Technology (ASSET'00), 2000, p. 25.

[43] M. Min, F. Wang, D.-Z. Du, P. M. Pardalos, A reliable virtual backbone scheme in mobile ad-hoc networks, in: Proc. of IEEE International Conference on Mobile Ad-hoc and Sensor Systems, 2004.

[44] H. Liu, R. Gupta, Selective backbone construction for topology control, in: Proc. of IEEE International Conference on Mobile Ad-hoc and Sensor Systems, 2004.

[45] R. Zheng, G. He, I. Gupta, L. Sha, Time idexing in sensor networks, in: Proc. of IEEE International Conference on Mobile Ad-hoc and Sensor Systems, 2004.

[46] O. Kachirski, R. Guha, Intrusion detection using mobile agents in wireless ad hoc networks, in: Proceedings of IEEE Workshop on Knowledge Media Networking, 2002.

[47] P.-J. Wan, X.-Y. Li, and O. Frieder, OVSF-CDMA Code Assignment in Wireless Ad Hoc Networks, in: Proc. of ACM International Workshop on Discrete Algorithms and Methods for Mobile Computing and Communications, 2004.

[48] O. Egecioglu, T. Gonzalez, Minimum-energy broadcast in simple graphs with limited node power, in: Proc. IASTED Int. Conf. on Parallel and Distributed Computing and Systems, 2001, pp. 334-338.

[49] X. Cheng, B. Narahari, R. Simha, M. X. Cheng, D. Liu, Strong minimum energy topology in wireless sensor networks: NP-completeness and heuristics, IEEE Transactions on Mobile Computing 2 (3).

[50] R. Zheng, J. C. Hou, L. Sha, Asynchronous wakeup for ad hoc networks, in: MobiHoc '03: Proc. of the 4th ACM international symposium on Mobile ad hoc networking \& computing, ACM Press, 2003, pp. 35-45.

[51] S. Parthasarathy, R. Gandhi, Distributed algorithms for coloring and domination in wireless ad hoc networks, in: Proc. of Conf. on Found. of Software Technology and Theoretical Computer Science (FSTTCS'04), 2004.

[52] Y. Wu, Q. Zhang, W. Zhu, S.-Y. Kung, Bounding the power rate function of wireless ad hoc networks, in: Proc. of IEEE INFOCOM, 2005.

[53] X. Cheng, X. Huang, D. Li, D.-Z. Du, Polynomial-time approximation scheme for minimum connected dominating set in ad hoc wireless networks, Networks 42 (4) (2003) 202-208. 


\section{A Appendix: Notations Used}

For easy reading, we summarize the notations used in the following table.

\begin{tabular}{|c|c|}
\hline $\mathcal{D}$ & a set of 2-dimensional interference disks \\
\hline$D_{i}$ & an interference disk $D\left(v_{i}, r_{v_{i}}\right)$ with index $i$ \\
\hline$l\left(D_{i}\right)$ & the level of a disk $D_{i}$ \\
\hline$v_{i}$ & the center of a disk $D_{i}$ \\
\hline$t_{i}$ & the transmission radius of wireless node $i$ \\
\hline$r_{i}$ & the radius of the interference disk $D_{i}$ \\
\hline$I(u, v)$ & the intersection region defined by two nodes $u$ and $v$ \\
\hline$k$ & parameter to control the quality of solution \\
\hline$\ell+1$ & the total levels of disks \\
\hline $\mathcal{D}(r, s)$ & disks that are active for shifting $(r, s)$ \\
\hline$M W I S(S, I)$ & $\begin{array}{l}\text { the maximum weighted set of independent disks inside a square } \\
S \text { and are independent with disks in } I\end{array}$ \\
\hline$V C(S, P)$ & $\begin{array}{l}\text { the minimum weighted set of disks such that } V C(S, P) \cup P \text { covers } \\
\text { all disks (with level at least } j \text { ) inside a } j \text {-square } S\end{array}$ \\
\hline$U_{S}$ & the set of disks in $U$ that are active for a $j$-square $S$ \\
\hline$U_{S}^{<j}$ & $\begin{array}{l}\text { the set of disks in } U \text { with level less than } j \text { that are active for } S \text {. } \\
\text { Similarly we define } U_{S}^{\leq j}, U_{S}^{=j}, U_{S}^{>j} \text {, and } U_{S}^{\geq j} \text {. }\end{array}$ \\
\hline$R$ & the set of active disks with level $\leq j$, intersecting a $j$-square $S$ \\
\hline$J$ & $\begin{array}{l}\text { a subset of independent active disks with level at most } j \text {, inter- } \\
\text { secting a } j \text {-square } S\end{array}$ \\
\hline$I$ & $\begin{array}{l}\text { the set of independent active disks in } J \text { with level less than } j \\
\text { that intersect a } j \text {-square } S\end{array}$ \\
\hline$Q$ & $\begin{array}{l}\text { the set of active disks not in } J \text {, with level at most } j \text {, intersecting } \\
\text { a } j \text {-square } S \text {. So } Q=R-J \text {. }\end{array}$ \\
\hline$P$ & $\begin{array}{l}\text { the set of active disks not in } J \text {, with level less than } j \text {, intersecting } \\
\text { a } j \text {-square }\end{array}$ \\
\hline$C, C_{2}$ & $\begin{array}{l}\text { upper-bounds on the number of independent active disks with } \\
\text { level at most } j \text { that intersect a } j \text {-square }\end{array}$ \\
\hline
\end{tabular}

